\title{
Assessing the Validity and Reliability of a Socio- Cognitive Determinants of Water Intake Questionnaire in Kidney Stones Patients
}

\section{Mehdi Mirzaei-Alavijeh}

Kermanshah University of Medical Sciences

Farzad Jalilian ( $\sim$ f_jalilian@yahoo.com)

Kermanshah University of Medical Sciences https://orcid.org/0000-0002-5923-7016

Halimeh Fatahi

Kermanshah University of Medical Sciences

Maryam Babakhani

Kermanshah University of Medical Sciences

\section{Laleh Solaimanizadeh}

Bam University of Medical Sciences

Research article

Keywords: Questionnaire, Health Education, Psychometric

Posted Date: July 30th, 2019

DOl: https://doi.org/10.21203/rs.2.12175/v1

License: (9) This work is licensed under a Creative Commons Attribution 4.0 International License. Read Full License 


\section{Abstract}

Background Developing a valid and reliable questionnaire is an important step in field studies, which can help to tailor health-related behavior interventions. This study aimed at the psychometric analysis of the socio-cognitive determinants of water intake questionnaire behaviors among patients with kidney stones in the west of Iran. Methods Items were generated from interviews with kidney stones patients and experts. As well as, our items pool were developed from the questionnaires of socio-cognitive determinants related to the water intake. Internal consistency, face, content, and construct validities were tested. Data were analyzed by SPSS (ver. 20.0). Results Based on Eigen values of $\geq 1.00$ and factor loadings of $\geq 0.40$, five determinants were extracted. The calculated Kaiser-Meyer-Olkin (KMO) value was 0.697 . The socio-cognitive determinants of water intake questionnaires were found to have acceptable internal consistency (Cronbach alpha of determinants between 0.65-0.85) and the theoretical assumptions for face, content, and construct validities were confirmed. Overall, the five studied sociocognitive determinants explained $73.83 \%$ of the variance in the proposed model. Conclusions The sociocognitive determinants of water intake questionnaire were revealed to have an acceptable psychometric evaluation. The questionnaire could be used to predict or explain water intake behavior in order to develop programs to increase water intake behavior among kidney stones patients.

\section{Background}

Kidney stone as the painful urologic disorder is the most common disorder of the urinary tract. [1] The prevalence of kidney stones increasing gradually, due to changes in diet (high fat, salt, animal protein diet), obesity, and a lack of physical activity, and generally in lifestyle. [2],[3] The kidney stone is a solid crystal formed from dissolved minerals in urine, may be caused by infections, environmental and metabolic factors; calcium oxalate $(\mathrm{C} 2 \mathrm{H} 2 \mathrm{CaO} 5)$ and/or phosphate stones accounted for $80 \%$ of all kidney stones. [4] Signs and symptoms of the kidney stones consisting; hematuria (blood in the urine), blockage of urine flow, kidney colic, urinary tract infections, flank pain, and or nausea/vomiting; In addition, kidney stones have been associated with an increased risk of major chronic illness such as cardiovascular diseases etc. [5] Kidney stone affects approximately $12 \%$ of the world population in their lifetime. [6] Importantly, the recurrence rate of kidney stones in Iran, for instance, is $32 \%$ after 5 years and $53 \%$ after a 10-year period. [7] Most of the patients diagnosed by kidney stones are middle-ages, around 30 to 60 years; both genders may be affected by kidney stones; although, men are the most affected, about $10-20 \%$ of men, and 3-5\% of women. [8] Due to the complications of kidney stones, prevention of kidney stones relapse is necessary and previous studies showed that inadequate intake of water is associated with more occurrences of kidney stones. [9] Moreover, increased water intake is a cost-benefits prevention strategy for recurrent kidney stones, based on evidence. [10] According to the American Urological Association Medical Management of Kidney Stone Guidelines, stone former should have a minimum urine output of $2.5 \mathrm{~L}$, daily. [11] Despite this cost-benefits prevention strategy, patients are seldom able to intake an adequate fluid in clinical practice. [10],[11] In health promotion programs research, it would be useful to know how socio-cognitive related determinants, such as attitude, 
subjective norms or self-efficacy are responsible to explain health-related behaviors. [12],[13],[14] Moreover, developing valid and reliable questionnaires is critical for researchers in order to apply for development successful promotion programs. [15] Thus, the basic socio-cognitive determinants should be identified in order to formulate intervention towards fluids intake among kidney stone patients, appropriately. [16],[17],[18],[19],[20] According to the absence of studies in developing countries, our study focused on assessing the initial validity and reliability of a socio-cognitive determinants questionnaire related to water intake in a sample of kidney stone patients in Iran.

\section{Methods}

\section{Item Generation}

The water intake questionnaire for kidney stones patients was designed to evaluate cognitive determinants that explain water intake behavior among kidney stones patients. Items were at first generated according to the consulting with 38 kidney stones patients and 12 experts, such as health educator and promoter, nephrology, health policy maker, health care management, nurses, and renal dieticians. As well as, our items pool were developed from the questionnaires of socio-cognitive determinants related to the water intake. [16],[17],[18],[19],[20] Interviews with kidney stones patients and experts were collected by one MSc health education and promotion student in a face-to-face interview style (15-25 min). After explaining the study's subjects, the participants signed consent forms, were interviewed separately by the interviewer. Plus, the interviewers had been trained to ensure that the participants completely realized their words.

The socio-cognitive determinants related to the water intake including; 23 items that measured the five constructs of 1) severity, 2) attitude, 3) subjective norms, 4) self-efficacy, and 5) barriers. Specifically, three items measured the perceived severity (e.g. "Inadequate water intake increases the relapse of kidney stones."). Four items were designed to measure a positive attitude towards water intake (e.g. "Water intake decreases the relapse of kidney stones."). Five items were measured subjective norms encouraging for water intake (e.g. "My family encourages me to drinking water"). Five items measured self-efficacy towards water intake (e.g. "I can drink water before feeling thirsty."). In addition, six items measured the perceived barriers (e.g. "I don't drink water if I don't feel thirsty."). In order to facilitate subjects responses to the items, all items were scored based on a five-point Likert scale, ranging from 1 (strongly disagree) to 5 (strongly agree).

\section{Validating the Questionnaire: Use of Face, Content and Construct Validities}

The validity of the questionnaire has been evaluated using face validity, content validity, and constructs validity.

Face Validity 
The qualitative method was used for evaluating face validity. In order to evaluate the face validity, a panel of 12 experts, such as health educator and promoter, nephrology, health policy maker, health care management, nurses, and renal dieticians were face-to-face interviewed to approve the difficulty, relevancy, clarity, and ambiguous of the questionnaire. Comments from the expert group were taken and minor modifications were made to some of the items based on expert opinions.

\section{Content validity}

Content Validity Ratio (CVR) and Content Validity Index (CVI) were used to assess content validity. [21] For this purpose, the questionnaire was given to a panel of 12 experts, including from six health educators, two health policymakers, two psychologists, and two urologists. Experts were asked to score each item by the following rankings as "completely necessary", "useful but unnecessary", and "unnecessary". So, the CVR was calculated using the "necessity" and "total item scores". To assess CVI, experts were asked to answer the following question, "did the items measure what they were intended to measure?" using a 4-point Likert scale in order to evaluate relevancy. A CVI value of each item was computed by dividing the number of experts who rated it as content valid (a ranking of 3 or 4 ) to the total number of experts. The total CVI value was calculated by dividing the sum of the " 3 " and " 4 " scores from each expert to the total number of experts. According to the Lawshe table the minimum value for acceptable CVR and CVI were considered 0.62 and 0.79 , respectively. [22]

\section{Construct Validity}

To assess the construct validity, firstly, the Classical Item Analysis (CIA) was used. In the CIA method, the mean, standard deviation, and Corrected Item-Total Correlation (CITC) of each item were calculated.

Secondly, the Exploratory Factor Analysis (EFA) with VARIMAX rotation using factor loadings of 0.40 was used to allocate items and to determine the degree which this factor structure replicated the original. The Kaiser-Meyer-Olkin (KMO) measured the sampling adequacy. The factorability of items was evaluated using the Bartlett test. Scree plot was used to confirm the strengths of the exploratory agents. Likewise, to determine agents, the values were equal or greater than 1.3 was considered. [23] The EFA evaluated by conducting a cross-sectional study of 115 kidney stone patients during 2018. Participants selected based on a convenience sampling method among kidney stone patients who referred to Imam Reza Hospital $(\mathrm{IRH})$ in the west of Iran. Only the subjects who diagnosed with the kidney stone were eligible to participate in this study. In addition, participants with incomplete personal or medical information and/or did not formally consent to participate, were excluded. Among the 115 kidney stone patients, 100 patients signed the consent form and voluntarily agreed to participate in our study. Therefore, the response rate was $86.9 \%$.

\section{Internal Consistency}

The reliability of the questionnaire was assessed by examining its internal consistency via Cronbach's alpha. Considering Cronbach's alpha, a threshold of 0.70 was acceptable. 
All data analysis was performed using the statistical package for social sciences (SPSS) (Version 20.0; IBM Corporation, Chicago, USA). A probability value ( $p$-value) of less than 0.05 was considered statistically significant.

\section{Results}

The mean age of respondents was 45.35 years, ranged from 20 to 70 years. Our findings indicated, $29 \%$ of participants were female and $71 \%$ of them were male. About $21 \%, 36 \%, 21 \%$, and $22 \%$ of the respondents reported had illiterate, primary/guidance school, high school/diploma, and academic education, respectively. Nearly $65 \%$ of them reported form the economically are independent. As well as $41 \%, 16 \%, 9 \%, 12 \%$ and $22 \%$ of the respondents reported business man, employed, retired, jobless, and housewife, respectively. In addition, $88 \%$ of them reported had health insurance. Furthermore, $74 \%$ of them reported living in urban and $26 \%$ of them reported living in rural.

The item impact method of all the sentences was more than 1.5 and, so; none of the twenty three items were omitted. Based on Lawshe's table, the acceptable lower limit for the CVR have been considered 0.62 and for CVI was 0.79 .Indicating content validity ratio and index for the all items was acceptable. In the CIA method, we found that one item from attitude construct, three items from attitude construct perceived barrier, one item from perceived self-efficacy construct, and one item from subjective norms construct had CITC less than 0.40 , and were deleted (Table 1). Therefore, eighteen finalized items were applied for the explanatory and confirmatory factor analysis.

Table 1: Items deleted in CIA (Supplementary Files)

In addition, as can be seen in Table 2, estimates of the reliability by using Cronbach's coefficient alpha, and our result showed the reliability coefficient for the all cognitive variables suggested that the internal consistency was adequate. The seventeen items were factor analyzed. The KMO test, which is the efficiency index of the sampling, was measured at 0.697. Bartlett's Test was also significant $(P<0.001)$ which indicated the data are suitable for the factorial analysis. Based on Eigen values of $\geq 1.00$ and factor loadings of $\geq 0.40$, five factors were extracted, accounting for $73.83 \%$ of the variation. More details of exploratory factor analysis are shown in Table 2. Furthermore, the scree plot diagram of factors is shown in Figure 1.

Table 2: Obtained result of the exploratory factor analysis (Supplementary Files)

\section{Discussion}

The aim of current study was to determine the psychometric evaluation of socio-cognitive determinants of water intake questionnaire among kidney stones patients. Our finding suggested that the sociocognitive determinants of water intake questionnaires were found to have acceptable internal 
consistency (Cronbach alpha of determinants between 0.65-0.85) and the theoretical assumptions for face, content, and construct validities were confirmed. This result is similar to the results reported by other studies. For example, Lindberg and Fernandes carried out research on 113 Portuguese hemodialysis patients with the aim of assessment fluid intake appraisal inventory and indicated the psychometric evaluation of the questionnaire is acceptable. [18] As well as, Arya et al showed their questionnaire for measuring the fluid intake, output, behavior, and urinary symptoms are valid and reliable. [19] In addition, Crary et al carried out the study with aim of psychometric analysis of a functional oral intake questionnaire for stroke patients and indicated this questionnaire had adequate reliability, validity, and sensitivity to change in functional oral intake. [24] Our findings suggested that our questionnaire has adequate reliability and validity. Our questionnaire could be used to predict or explain water intake behavior in order to develop programs to increase water intake behavior among kidney stones patients. A benefit to using this tool is that the information obtained can be used in clinical practice to tailor interventions program for patients with kidney stones. Although this instrument does not address the full range of cognitive determinant, it does reflect patients' self-efficacy, attitude, subjective norms, perceived severity, and barriers.

Moreover, the perceived severity, attitude, subjective norms, self-efficacy, and perceived barriers were predicted the greater variance of the hypothesized model, respectively. Several study indicated cognitive constructs such as attitude, self-efficacy, barrier, perceived severity and subjective norms, were associated with the chronic patients to uptake healthy behaviors. [16],[17] For example, McCauley et al in their study application the health belief model to determine the cognitive variables on fluid intake behavior among kidney stone formers and indicated some support to use the HBM to design and implement interventions to modify or improve individuals' beliefs that kidney stone patients may improve fluid intake behavior. [16]

Our sample included kidney stones patients in the west of Iran. Nevertheless, representatives of other populations were not available in the current study. In addition according to the non-probability of data collection, our findings may be not generalized to other groups of kidney stone patients. Not evaluation of the external validity of questionnaire was another limitation in our study. Future studies to evaluation external validity are necessary.

\section{Conclusion}

Design evidence-based intervention to kidney stone patients in order to increase water intake is important. Our study has provided significant information for health planning programmers to develop water intake promotion programs among kidney stone patients. In other words, the use of our scale may be useful for guiding implementers to evaluation the determinants related to water intake and the development of effective health promotion programs among Iranian kidney stone patients. In general, we have found that using the current questionnaire helps us to identify the predictors of low water intake in patients with kidney stones. Knowing exactly where the problems with water intake occur can be helpful in focusing interventions for the patient with kidney stones. Finally, the validity and reliability of the instrument 
proposed for the cognitive constructs are adequate to support using this questionnaire in research of prediction of water intake among Iranian kidney stones patients.

\section{Abbreviations}

\begin{tabular}{ll} 
CIA & Classical Item Analysis \\
\hline CITC & Corrected Item-Total Correlation \\
\hline CVI & Content Validity Index \\
\hline CVR & Content Validity Ratio \\
\hline EFA & Exploratory Factor Analysis \\
\hline KMO & Kaiser-Meyer-Olkin \\
\hline IRH & Imam Reza Hospital \\
\hline SPSS & Statistical Package for Social Sciences
\end{tabular}

\section{Declarations}

\section{Ethics approval and consent to participate}

The research ethics committee at deputy of research of the Kermanshah University of Medical Sciences in the west of Iran had approved the study protocol (KUMS.REC.1397.045). Further, the participants had been given the participant information statement, and had signed the written consent form. Individual personal information was kept confidential.

\section{Consent for publication}

Not applicable.

\section{Availability of data and material:}

Please contact the corresponding author for data requests.

\section{Competing interests}

The authors have no conflicts of interest to declare.

\section{Funding}

This study was funded by the Kermanshah University of medical sciences in the west of Iran. The funding organization has no role in the design of the study, collection, analysis, and interpretation of data and in writing the manuscript this was the role of authors 


\section{Authors' contributions}

MMA and FJ contributed to the conception and design of the research; HF, MB and LS contributed to the acquisition design; MMA and FJ contributed to the analysis and interpretation of the data. All authors approved the final manuscript.

\section{Acknowledgements}

The authors would like to thank Kermanshah University of Medical Sciences, Kermanshah, Iran.

\section{References}

1. López M, Hoppe B. History, epidemiology and regional diversities of urolithiasis. Pediatric nephrology. 2010; 25(1):49.

2. Deepika RM, Ravisankar P, Priya JD, Surekha P, Sushmitha G, Tejaswi V, Ramesh G. Renal Calculi: A Comprehensive Review. Indian Journal of Research in Pharmacy and Biotechnology. 2018; 6(1):30-4.

3. Mikawlrawng K, Kumar S, Vandana R. Current scenario of urolithiasis and the use of medicinal plants as antiurolithiatic agents in Manipur (North East India): a review. International Journal of Herbal Medicine. 2014; 2(1):1-2.

4. Coe FL, Evan A, Worcester E. Kidney stone disease. The Journal of clinical investigation. 2005; 115(10):2598-608.

5. Rule AD, Roger VL, Melton LJ, Bergstralh EJ, Li X, Peyser PA, Krambeck AE, Lieske JC. Kidney stones associate with increased risk for myocardial infarction. Journal of the American Society of Nephrology. 2010; 21(10):1641-4.

6. Chauhan CK, Joshi MJ, Vaidya AD. Growth inhibition of struvite crystals in the presence of herbal extract Commiphora wightii. Journal of Materials Science: Materials in Medicine. 2009; 20(1):85.

7. Safarinejad MR. Adult urolithiasis in a population-based study in Iran: prevalence, incidence, and associated risk factors. Urological research. 2007; 35(2):73-82.

8. Edvardsson VO, Indridason OS, Haraldsson G, Kjartansson O, Palsson R. Temporal trends in the incidence of kidney stone disease. Kidney international. 2013; 83(1):146-52.

9. Dhanalakshmi S, Gayathri R, Vishnupriya V. Correlation between Inadequate Intake of Water and Prevalence of Renal Calculi-A Survey. Journal of Pharmaceutical Sciences and Research. 2017;9(4):429

10. Lotan Y, Buendia Jiménez I, Lenoir-Wijnkoop I, Daudon M, Molinier L, Tack I, Nuijten MJ. Primary prevention of nephrolithiasis is cost-effective for a national healthcare system. BJU international. 2012; 110(11c):E1060-7.

11. Pearle MS, Goldfarb DS, Assimos DG, Curhan G, Denu-Ciocca CJ, Matlaga BR, Monga M, Penniston $\mathrm{KL}$, Preminger GM, Turk TM, White JR. Medical management of kidney stones: AUA guideline. The Journal of urology. 2014; 192(2):316-24. 
12. Takaki J, Yano E. Possible gender differences in the relationships of self-efficacy and the internal locus of control with compliance in hemodialysis patients. Behavioral Medicine. 2006; 32(1):5-11.

13. O'Connor SM, Jardine AG, Millar K. The prediction of self-care behaviors in end-stage renal disease patients using Leventhal's Self-Regulatory Model. Journal of Psychosomatic Research. 2008; 65(2):191-200.

14. Rich A, Brandes K, Mullan B, Hagger MS. Theory of planned behavior and adherence in chronic illness: a meta-analysis. Journal of behavioral medicine. 2015;38(4):673-88.

15. Barua A. Methods for decision-making in survey questionnaires based on Likert scale. Journal of Asian Scientific Research. 2013; 3(1):35-8.

16. McCauley LR, Dyer AJ, Stern K, Hicks T, Nguyen MM. Factors influencing fluid intake behavior among kidney stone formers. The Journal of urology. 2012; 187(4):1282-6.

17. Khalil A, Abdalrahim M. Knowledge, attitudes, and practices towards prevention and early detection of chronic kidney disease. International nursing review. 2014;61(2):237-45.

18. Lindberg M, Fernandes MA. self-efficacy in relation to limited fluid intake amongst Portuguese haemodialysis patients. Journal of renal care. 2010; 36(3):133-8.

19. Arya LA, Banks C, Gopal M, Northington GM. Development and testing of a new instrument to measure fluid intake, output, and urinary symptoms: the questionnaire-based voiding diary. American journal of obstetrics and gynecology. 2008; 198(5):559-e1

20. Lindberg M, Wikström B, Lindberg P. Fluid Intake Appraisal Inventory: Development and psychometric evaluation of a situation-specific measure for haemodialysis patients' self-efficacy to low fluid intake. Journal of psychosomatic research. 2007; 63(2):167-73.

21. Polit DF, Beck CT, Owen SV. Is the CVI an acceptable indicator of content validity? Appraisal and recommendations. Research in nursing \& health. 2007; 30(4):459-67.

22. Lawshe $\mathrm{CH}$. A quantitative approach to content validity 1. Personnel psychology. 1975; 28(4):563-75.

23. Hayton JC, Allen DG, Scarpello V. Factor retention decisions in exploratory factor analysis: A tutorial on parallel analysis. Organizational research methods. 2004; 7(2):191-205.

24. Crary MA, Mann GD, Groher ME. Initial psychometric assessment of a functional oral intake scale for dysphagia in stroke patients. Archives of physical medicine and rehabilitation. 2005; 86(8):1516-20.

\section{Tables}

Due to technical limitations, tables are only available as a download in the supplemental files section.

\section{Figures}




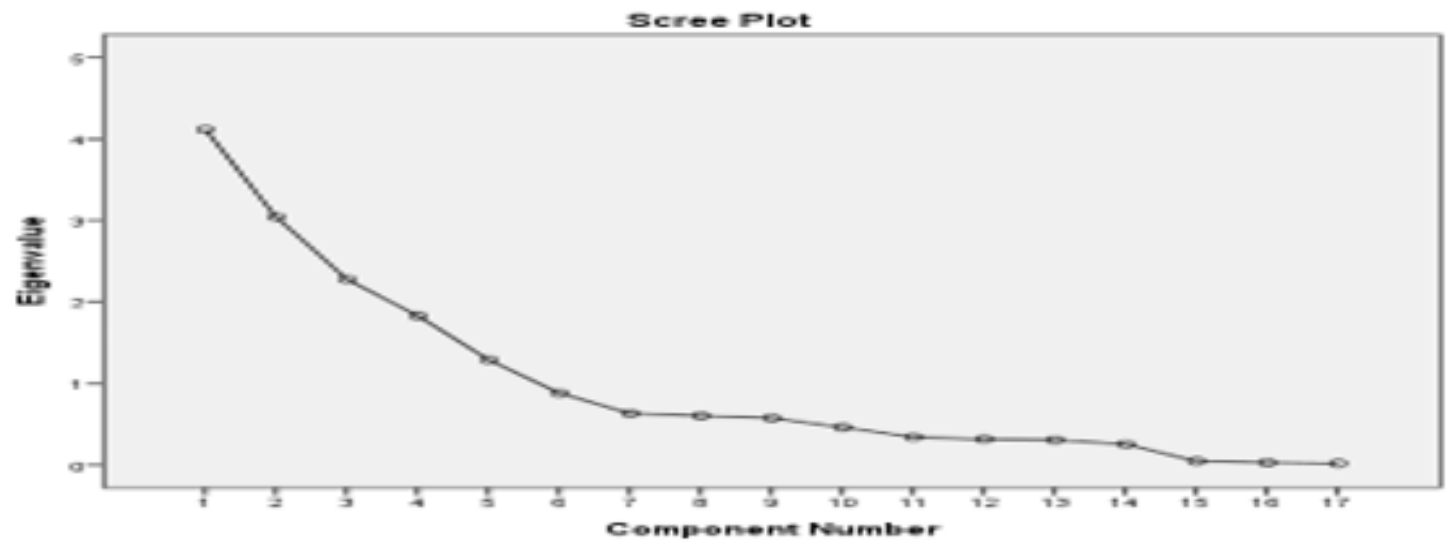

Figure 1: The scree plot of the structures studied among the participants.

The scree plot diagram of factors is shown in Figure 1.

\section{Figure 1}

The scree plot of the structures studied among the participants. The scree plot diagram of factors is shown in Figure 1.

\section{Supplementary Files}

This is a list of supplementary files associated with this preprint. Click to download.

- supplement1.jpg

- supplement2.jpg 